\title{
Overexpression of Dyrk1A Causes the Defects in Synaptic Vesicle Endocytosis
}

\author{
Yoonju Kim ${ }^{\mathrm{a}, \mathrm{b}, \mathrm{e}}$ Joohyun Park ${ }^{\mathrm{a}-\mathrm{c}}$ Woo-Joo Song ${ }^{\mathrm{d}}$ Sunghoe Chang ${ }^{\mathrm{a}-\mathrm{c}}$ \\ ${ }^{a}$ Department of Physiology and Biomedical Sciences, ${ }^{\text {b }}$ Neuroscience Research Institute, Medical Research Center, \\ and ${ }^{\mathrm{C} B i o m e m b r a n e}$ Plasticity Research Center, Seoul National University College of Medicine, Seoul, dinstitute for \\ Brain Science and Technology (IBST), FIRST Research Group, Inje University, Busan, and e School of Life Science, \\ Gwangju Institute of Science and Technology, Gwangju, South Korea
}

\section{Key Words}

Dyrk1A $\cdot$ Down syndrome $\cdot$ Clathrin-mediated endocytosis · Synaptic vesicle $\cdot$ Phosphorylation .

Endocytic proteins gest that aberrant Dyrk1A-mediated phosphorylation of the endocytic machinery perturbs synaptic vesicle endocytosis, which may contribute to synaptic dysfunctions and cognitive deficits associated with DS.

Copyright $\odot 2010$ S. Karger AG, Basel

\begin{abstract}
Trisomy 21-linked Dyrk1A (dual-specificity tyrosine phosphorylation-regulated kinase $1 \mathrm{~A}$ ) overexpression is implicated in pathogenic mechanisms underlying mental retardation in Down syndrome (DS). It is known to phosphorylate multiple substrates including endocytic proteins in vitro, but the functional consequence of Dyrk1A-mediated phosphorylation on endocytosis has never been investigated. Here, we show that overexpression of Dyrk1A causes defects in clathrin-mediated endocytosis and specifically, in the recruitment of endocytic proteins to clathrin-coated pits in fibroblasts. Synaptic vesicle endocytosis also significantly slowed down as a result of Dyrk1A overexpression in cultured hippocampal neurons. These effects are dependent on Dyrk1A kinase activity. The inhibitory effect of Dyrk1A on synaptic vesicle endocytosis was confirmed in neuronal cultures derived from transgenic mice overexpressing Dyrk1A at levels found in DS. Pharmacological blockade of Dyrk1A with epigallocatechin gallate rescued the endocytic phenotypes found in transgenic neurons. Together, our results sug-
\end{abstract}

\section{Introduction}

Down syndrome (DS), also referred to as trisomy 21 , is the most common genetic cause of mental retardation with an incidence of 1 in every $\sim 700$ births. DS is associated with a complex variety of phenotypic manifestations, including cognitive deficits, hypotonia, congenital heart defects and immune system anomalies [1]. Although DS is a multigenic disorder, evidence suggests that the overexpression of specific genes or small groups of genes may contribute significantly to some DS-associated phenotypes. DYRK1A (dual-specificity tyrosine phosphorylation-regulated kinase 1A), which encodes a proline-directed serine/threonine kinase and maps to the DS critical region (DSCR) of human chromosome 21, is one such gene. Recent studies have reported that the protein levels as well as the kinase activity of Dyrk1 A are both increased by $\sim 1.5$-fold in DS brains relative to controls [2]. Importantly, Dyrk1A overexpression in transgenic mice has

\section{KARGER}

Fax +4161306 1234

E-Mail karger@karger.ch

www.karger.com (c) 2010 S. Karger AG, Basel

$1424-862 X / 10 / 0183-0164 \$ 26.00 / 0$

Accessible online at:

www.karger.com/nsg
Sunghoe Chang, $\mathrm{PhD}$

Department of Physiology, Seoul National University College of Medicine

309 Biomedical Science Bldg., 28 Yeongeon-dong, Jongno-gu

Seoul 110-799 (South Korea)

Tel. +82 2740 8918, Fax +82 23673 2167, E-Mail sunghoe@ snu.ac.kr 
been shown to cause cognitive impairment, while genetic disruption of Dyrk1A leads to seizures and developmental defects, including microcephaly and growth retardation. However, the molecular and cellular bases of these phenotypes are not fully understood $[1,3,4]$.

Although Dyrk1A contains a bipartite nuclear targeting sequence, a substantial pool of endogenous Dyrk1A is localized to the neuronal cytoplasm as well as at synapses in the brain $[5,6]$. Dyrk1A phosphorylates several endogenous substrates including multiple endocytic proteins such as dynamin, amphiphysin, endophilin and synaptojanin 1 [7-10]. Interestingly, synaptojanin 1 is a synapse-enriched phosphoinositide phosphatase that is encoded by SYNJ1, another gene that maps on human chromosome 21 [11]. Accordingly, SYNJ1 is overexpressed in DS [12] and transgenic models harboring higher levels of synaptojanin 1 exhibit cognitive deficits [13]. At synapses, endocytic proteins are phosphorylated in resting states but are coordinately dephosphorylated by calcineurin upon depolarization, which stimulates their assembly and promotes endocytosis $[14,15]$. Based on these findings as well as work on other kinases (e.g. Cdk5), it has been hypothesized that Dyrk1A may regulate the process of synaptic vesicle recycling by triggering the disassembly of the endocytic machinery. Additionally, overexpression of Dyrk1A may perturb the dynamics of endocytic protein assembly/disassembly at synapses, which, in turn, may contribute to synaptic malfunction and cognitive deficits in individuals with DS [14-16]. However, direct functional evidence for a role of Dyrk1A in endocytosis and synaptic vesicle endocytosis is still lacking.

Here we show that overexpression of Dyrk1A caused defects in clathrin-mediated endocytosis in fibroblasts as well as in neurons. Our results indicate that Dyrk1A-mediated phosphorylation of core endocytic proteins may significantly contribute to DS-linked synaptic malfunction. Since other chromosome 21-linked proteins, such as synaptojanin and RCAN1, are both implicated in endocytosis, their overexpression may produce additive effects on synaptic vesicle endocytosis and possibly contribute to cognitive impairment in DS.

\section{Materials and Methods}

\section{Materials}

His-tagged Dyrk1A was provided by Dr. Kwang Chul Chung (Yonsei University, Seoul, South Korea). The kinase dead mutant of Dyrk1A, 2YF (Y319F/Y321F), was generated by site-directed point mutations using the QuickChange ${ }^{\circledR}$ Site-Directed Mutagenesis Kit (Stratagene, Austin, Tex., USA). Dyrk1A and Dyrk1A-
2YF mutants were amplified by PCR and the PCR products were subcloned into mCherry vector (kindly provided by Dr. Roger Y. Tsien at UCSD, San Diego, Calif., USA). GFP-dynamin 2(baa), mRFP-clathrin light chain, GFP-amphiphysin 1 and GFP-endophilin 2 were kindly provided by Pietro De Camilli (Yale University, New Haven, Conn., USA). All DNA constructs were verified by DNA sequencing. Alexa fluor 488-transferrin was from Molecular Probes (Eugene, Oreg., USA). And all other reagents were from Sigma (St. Louis, Mo., USA).

\section{Transferrin Uptake Assay}

Forty-eight hours after transfection, COS-7 cells were starved for $4 \mathrm{~h}$ in serum-free DMEM with $20 \mathrm{mM}$ HEPES and incubated in serum-free DMEM with $20 \mathrm{mM}$ HEPES containing $10 \mu \mathrm{g} / \mathrm{ml}$ Alexa-fluor 488-transferrin (Alx488-Tfr) for 0, 1, 3, 5, 15 and 30 $\mathrm{min}$ at $37^{\circ} \mathrm{C}$. The cells were washed in acid stripping solution (150 $\mathrm{mM} \mathrm{NaCl}, 2 \mathrm{mM} \mathrm{CaCl}_{2}$, and $\left.25 \mathrm{mM} \mathrm{CH}_{3} \mathrm{COONa}, \mathrm{pH} 4.5\right)$ and fixed in $4 \%$ paraformaldehyde, $4 \%$ sucrose, PBS for $15 \mathrm{~min}$. We have manually selected the cells showing moderate levels of mCherry expression (A.F.U 200 to 350) and excluded the cells showing either low or high levels of mCherry expression. Images were taken on an Olympus IX-71 microscope (Olympus Corp., Tokyo, Japan) using a $\times 60,1.35$ N.A. objective lens and Hamamatsu OrcaII CCD camera (Hamamatsu Photonics, Hamamatsu City, Japan). Alx488-Tfr intensities were averaged over individual cells and analyzed using MetaMorph Imaging software (Universal Imaging Corp., West Chester, Pa., USA).

\section{Total Internal Reflection Fluorescence Microscopy}

A total internal reflection fluorescence microscope (TIRFM) employs the phenomenon of total internal reflection, which occurs at the interface between glass and aqueous solution. At large angle of incidence, the excitation laser beam reflects back into glass and evanescent wave is generated. The evanescent electromagnetic field decays exponentially from the interface, and thus penetrates to a depth of only approximately $100 \mathrm{~nm}$ into the sample medium. Therefore, only molecules that are at the TIRF surface are excited and fluoresce, while molecules at the distances larger than $100-200 \mathrm{~nm}$ are not excited and do not fluoresce. The selective visualization of surface regions immediately beneath the plasma membrane by TIRFM renders the features and events on the plasma membrane in living cells with high axial resolution. For imaging with TIRF microscope, medium was replaced with imaging buffer containing $136 \mathrm{mM} \mathrm{NaCl}, 2.5 \mathrm{mM} \mathrm{KCl}, 2 \mathrm{mM}$ $\mathrm{CaCl}_{2}, 1.3 \mathrm{mM} \mathrm{MgCl}_{2}, 10 \mathrm{mM}$ HEPES, and $10 \mathrm{mM}$ glucose at $\mathrm{pH}$ 7.4. The cells were imaged by TIRFM using an Olympus IX-71 inverted microscope fitted with a $\times 60,1.45$ N.A. TIRF lens and controlled by Cell ${ }^{\mathrm{TM}}$ software (Olympus Corp., Tokyo, Japan). Laser lines (488 and $561 \mathrm{~nm}$ diode lasers) were coupled to the TIRFM condenser through two independent optical fibers. The calculated evanescent depth was $\approx 100 \mathrm{~nm}$. Cells were typically imaged in two channels by excitation with 0.1 - to 0.2 -second exposures and detected with a back-illuminated Andor iXon887 EMCCD camera $(512 \times 512$, 16-bit; Andor Technologies, Belfast, UK). For analysis, we used Image J program (National Institutes of Health).

\section{Neuron Culture and Transfection}

Dyrk1A TG mice that overexpressed the human Dyrk1A gene, which was transcribed from the genomic Dyrk1A DNA carried 
on a bacterial artificial chromosome, were produced and maintained as described previously [3]. Experiments were performed in accordance with guidelines set forth by the Inje University Council Directive for the proper care and use of laboratory animals. Hippocampal neurons derived from E-18 primary rats or P1 Dyrk1A TG mice were prepared as described [17]. Briefly, hippocampi were dissected, dissociated with papain, and triturated with a polished half-bore Pasteur pipette. The cells $\left(2.5 \times 10^{5}\right)$ in minimum Eagle's medium supplemented with $0.6 \%$ glucose, $1 \mathrm{~mm}$ pyruvate, $2 \mathrm{~mm}$ L-glutamine, $10 \%$ fetal bovine serum, and antibiotics were plated on poly-D-lysine-coated glass coverslips in a $60-\mathrm{mm}$ Petri dish. Four hours after plating, the medium was replaced with basal media Eagle's (Invitrogen, San Diego, Calif., USA) supplemented with 2\% B-27, $10 \mathrm{~mm}$ HEPES, and $0.5 \mathrm{~mm}$ pyruvate or Neurobasal (Invitrogen) supplemented with 2\% B-27, $0.5 \mathrm{mM}$ L-glutamine. $4 \mu \mathrm{M}$ of $1-\beta$-D-cytosine-arabinofuranoside (Ara-C, Sigma) was added as needed. Neurons were transfected using calcium-phosphate method [18]. Briefly, vGlut1-pHluorin $(\mathrm{vGpH})$ with either mCherry, mCherry-Dyrk1A or its kinase dead mutant was transfected at 10 days in vitro. The vGpH and Dyrk1A constructs were cotransfected in a ratio of 1:3.

\section{vGlut1-pHluorin Endocytosis Assay}

Coverslips were mounted in a perfusion/stimulation chamber equipped with platinum-iridium field stimulus electrodes (EC-S10, LCI, Seoul, Korea) on the stage of an Olympus IX-71 inverted microscope with $\times 40,1.0$ N.A. oil lens. The cells were continuously perfused at room temperature with imaging buffer solution. $10 \mu \mathrm{M}$ 6-cyano-7-nitroquinoxaline-2,3-dione and $50 \mu \mathrm{M}$ of DL-2amino-5-phosphonovaleric acid were added to imaging buffer to reduce spontaneous activity and prevent recurrent excitation during stimulation. Time-lapse images were acquired every 10 or $5 \mathrm{~s}$ for 4 min using a Hamamatsu OrcaII CCD camera driven by MetaMorph. From the 4 th frame, the cells were stimulated ( $1 \mathrm{~ms}$, $20-50 \mathrm{~V}$, bipolar) for $60 \mathrm{~s}$ at $5 \mathrm{~Hz}, 30 \mathrm{~s}$ at $10 \mathrm{~Hz}$ or $15 \mathrm{~s}$ at $20 \mathrm{~Hz}$ using an A310 Accupulser current stimulator (World Precision Instruments, Sarasota, Fla., USA). Quantitative measurements of the fluorescence intensity at individual boutons were obtained by averaging a selected area of pixel intensities using Image J program (National Institutes of Health). Average intensities of individual regions were also calculated by Image J. Large puncta, typically representative of clusters of smaller synapses, were rejected during the selection procedure. Fluorescence was expressed in intensity units that correspond to fluorescence values averaged over all pixels within the region of interest. Light from a mercury lamp was shuttered using a VMM1 Unibilitz shutter (Vincent Associates, Rochester, N.Y., USA). Net fluorescence changes were obtained by subtracting the average intensity of the first four frames $\left(F_{0}\right)$ from the intensity of each frame $\left(F_{t}\right)$ for individual boutons. They were then normalized to the maximum fluorescence intensity $\left(F_{\max }-F_{0}\right)$ and averaged. The decay of fluorescence was fitted with a single exponential. All fitting was done using individual error bars to weight the fit, using SigmaPlot 8.0. In some experiments where fluorescence decay deviated from single exponential behavior, we obtained the best fitting single exponential function from the early portion of the decay. Data are presented as means \pm SE. Statistical analysis was carried out with PASW Statistics 18 (formerly SPSS Statistics). For multiple conditions, we compared means by analysis of variance (ANOVA) followed by Tukey's HSD post hoc test.

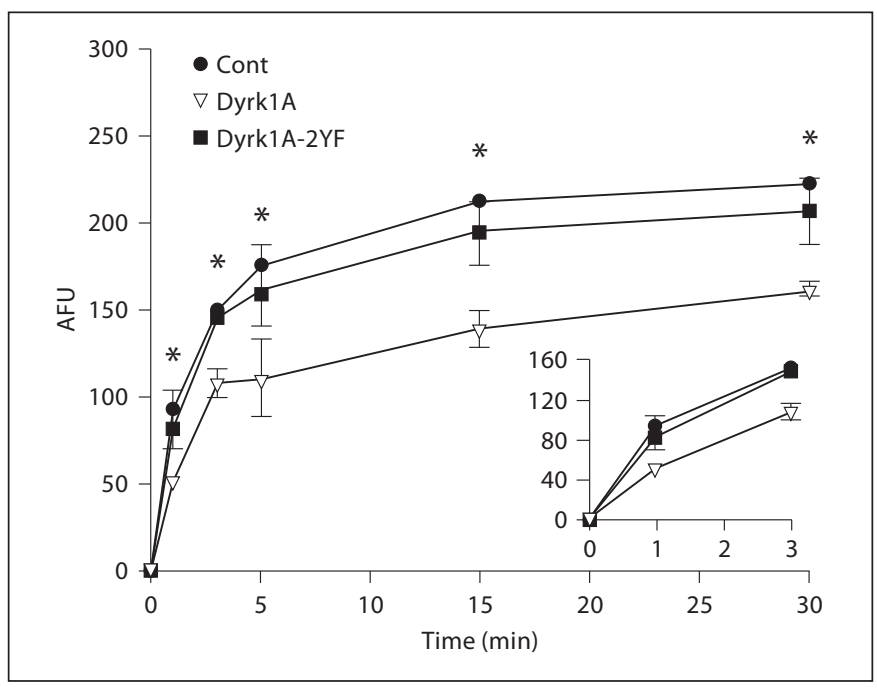

Fig. 1. Transferrin uptake is impaired by overexpression of Dyrk1A. COS-7 cells were transfected with mCherry (Cont), Dyrk1A and Dyrk1A-kinase dead mutant (Dyrk1A-2YF). $48 \mathrm{~h}$ after transfection, the cells were starved for $4 \mathrm{~h}$ and then incubated with Alexa fluor 488 -transferrin for indicated times at $37^{\circ} \mathrm{C}$. At each time point, the cells were fixed and imaged. 50 cells expressing mCherry fluorescence were analyzed at every time point for each group. Data are presented as means \pm SE of three independent experiments. Inset expands data collected within initial 3 min. ${ }^{*} \mathrm{p}<0.05$ (ANOVA and Tukey's HSD post hoc test).

\section{Results}

To test whether Dyrk1A affects clathrin-mediated endocytosis in general, we first overexpressed Dyrk1A in COS-7 cells and performed transferrin uptake experiment. COS-7 cells were transfected with the Dyrk1A, Dyrk1A-2YF (kinase-dead mutant) or control vector, and then allowed to take up Alexa fluor-488 conjugated transferrin (Alx488-Tfr) for the indicated times at $37^{\circ} \mathrm{C}$ (fig. 1). In control, Alx488-Tfr was internalized and accumulated in the perinuclear endosomal compartment and the uptake was reached at the plateau $\sim 25 \mathrm{~min}$ after incubation. In cells overexpressing Dyrk1A, however, the internalization was significantly delayed and there is $\sim 2$-fold decrease in the initial rate of transferrin uptake relative to the control (fig. 1, inset). The total amount of transferrin uptake after incubation in Dyrk1A overexpressing cells was $\sim 35 \%$ less than that in control. The overexpression of Dyrk1A-2YF did not cause any defects in the uptake of Alx488-Tfr, suggesting Dyrk1A-directed phosphorylation is attributed to its inhibitory effect on transferrin uptake.

The phosphorylation of key endocytic proteins such as dynamin, amphiphysin or endophilin is known to pre- 


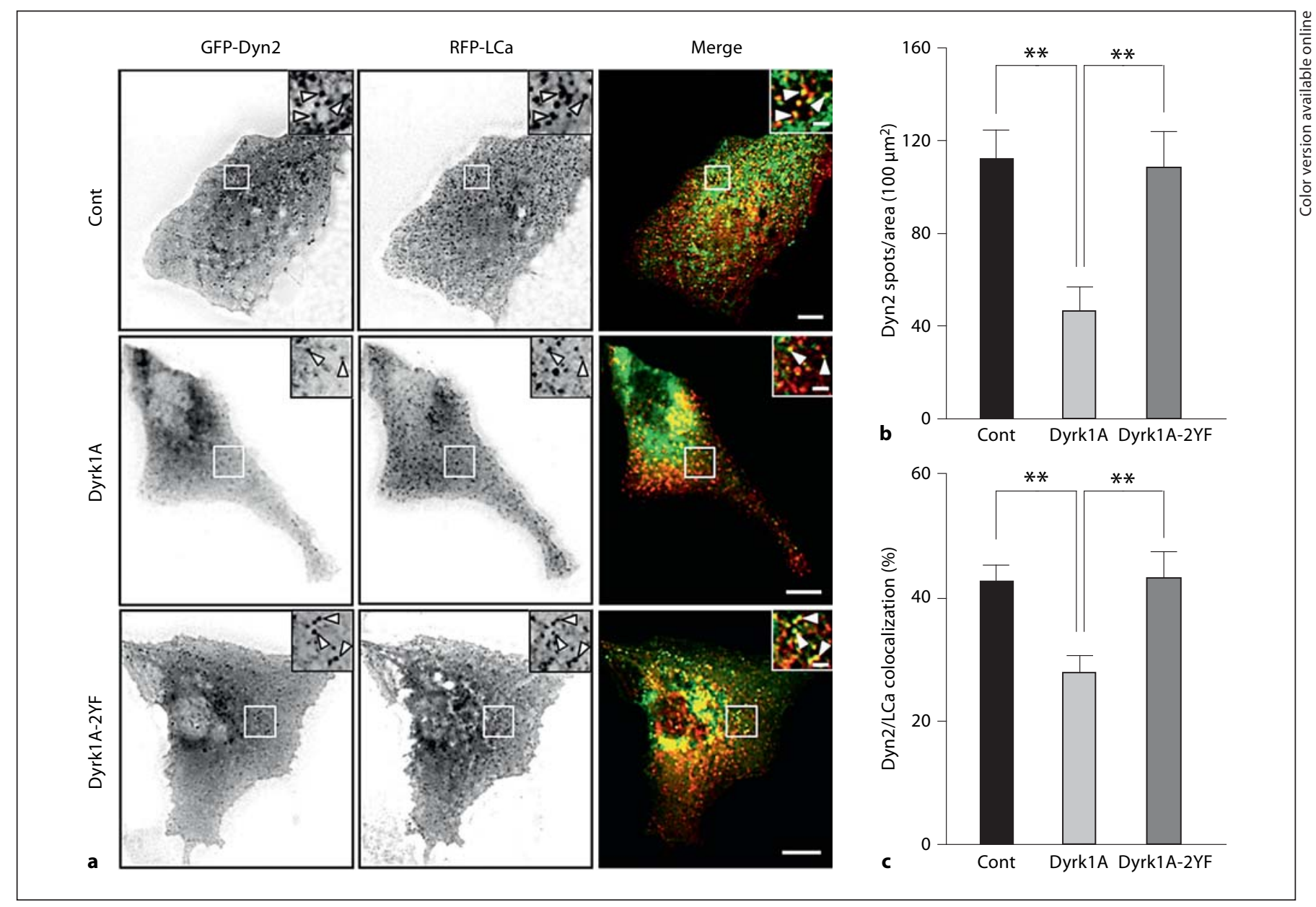

Fig. 2. The overexpression of Dyrk1A induces the defects in recruitment of dynamin 2 to the sites of clathrin-coated pit formation. a TIRFM images of COS-7 cells which were triply transfected with mRFP-clathrin-light chain (RFP-LCa), GFP-dynamin 2 (GFP-Dyn2), and Dyrk1A, Dyrk1A-2YF or pcDNA-His (Cont) vector. Arrowheads indicate the colocalization between Dyn 2 and LCa. Scale bars: low magnification, $10 \mu \mathrm{m}$; high magnification,
$2 \mu \mathrm{m}$. $\mathbf{b}$ The number of Dyn2 spots within $100 \mu \mathrm{m}^{2}$ of the plasma membrane. c The degree of colocalization between Dyn2 and LCa was reduced by Dyrk1A overexpression but not by Dyrk1A-2YF ( $\mathrm{n}=7$ for Cont, $\mathrm{n}=6$ for Dyrk1A, $\mathrm{n}=8$ for Dyrk1A2YF). 6-10 cells were selected in each independent experiment and were analyzed. Data are presented as means \pm SE. ${ }^{* *} \mathrm{p}<0.01$ (ANOVA and Tukey's HSD post hoc test). vent endocytic coat complexes from assembling [7-10, 14, 19]. Since Dyrk1A also phosphorylates these endocytic proteins, and its overexpression causes the defects in transferrin uptake, we tested whether Dyrk1A affects the processes of clathrin coat complex formation using a TIRFM, which allows us to selectively visualize the features and events on the plasma membrane in living cells with high axial resolution [20-22]. COS-7 cells were triply transfected with GFP-dynamin 2, mRFP-clathrinlight chain (LCa), and Dyrk1A, Dyrk1A-2YF or control vector. Like dynamin 1, dynamin 2 was phosphorylated by Dyrk1A (online supplementary figure 1, www. karger.com/doi/10.1159/000321994). We found that com- pared to control, Dyrk1A overexpression significantly decreased the number of dynamin 2 spots at the plasma membrane (fig. 2a, b). The colocalization between dynamin 2 and clathrin at the plasma membrane was also significantly reduced (fig. $2 \mathrm{a}, \mathrm{c}$ ), suggesting that less dynamin 2 was recruited to the sites of clathrin-coated pit formation upon Dyrk1A overexpression. The overall expression of dynamin 2 was not affected by Dyrk1A overexpression (online supplementary figure 2). Again, Dyrk1A-2YF failed to show any defects in dynamin 2 recruitment (fig. 2). The recruitment of amphiphysin 1 or endophilin 2 at the plasma membrane was also inhibited by overexpression of Dyrk1A (online supplementary figures 3 and 4). 

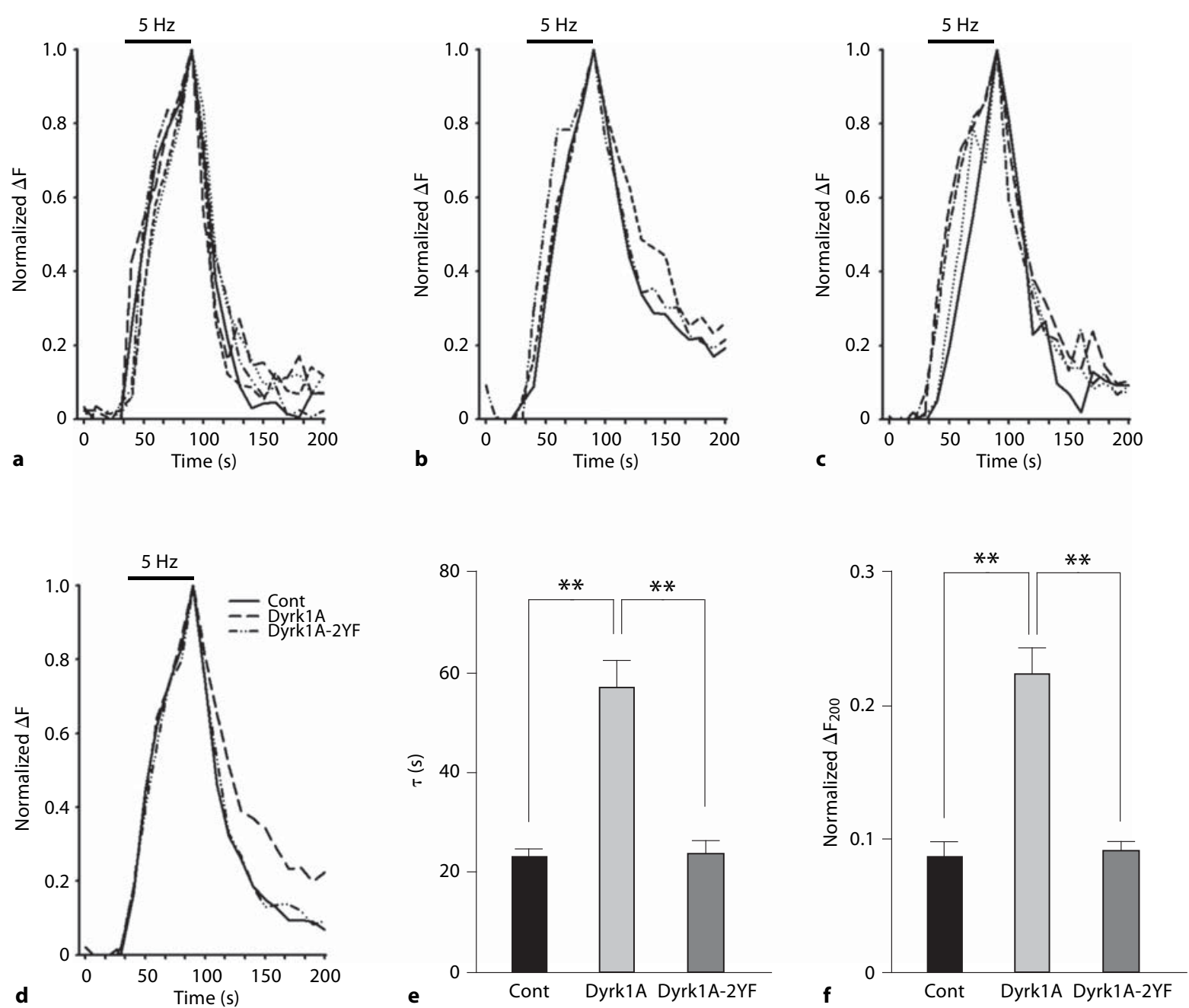

Fig. 3. The overexpression of Dyrk1A impairs synaptic vesicle endocytosis. Hippocampal neurons were cotransfected with vGpH, and mCherry (Cont), Dyrk1A or Dyrk1A-2YF. a-c Normalized $\mathrm{vGpH}$ fluorescence intensity profiles of the boutons from Cont (a), Dyrk1A (b), or Dyrk1A-2YF (c) overexpressing neurons which were stimulated with 300 APs at $5 \mathrm{~Hz}$. Each line (solid, dotted, etc.) represents an average fluorescence trace from a single neuron. 4-5 neurons were selected in each independent experiment and $10-20$ boutons from a single neuron were analyzed. $\mathbf{d}$ Average

Next, we tested the effect of Dyrk1A overexpression on synaptic vesicle endocytosis in neurons. Cultured hippocampal neurons were transfected with vGlut1-pHluorin $(\mathrm{vGpH})$ with various constructs, and stimulated with 300 action potentials (APs) at $5 \mathrm{~Hz}$. vGpH is a vesicular glutamate transporter 1 fused with pHluorin, a modified GFP with high $\mathrm{pH}$ sensitivity [23]. The relative basic $\mathrm{pH}$ of the extracellular space allows the $\mathrm{vGpH}$ to fluoresce fluorescence intensity profiles were plotted as $\Delta \mathrm{F} / \mathrm{F}_{0}$ against time, following stimulation with $300 \mathrm{APs}$ at $5 \mathrm{~Hz}$ (dark bar). Data were collected from 3 to 5 independent experiments for each group. e The decay of $\mathrm{vGpH}$ fluorescence was fitted by a single exponential with $\tau=23.0 \pm 1.8 \mathrm{~s}$ for control; $\tau=56.7 \pm 5.7 \mathrm{~s}$ for Dyrk1A; $\tau=23.7 \pm 2.5 \mathrm{~s}$ for Dyrk1A-2YF. $\mathbf{f}$ The residual vGpH fluorescence intensity at $200 \mathrm{~s}$ was normalized to the initial resting state of each presynaptic bouton. Data are presented as means \pm SE. ${ }^{* *} \mathrm{p}<0.01$ (ANOVA and Tukey's HSD post hoc test).

and its fluorescence is quenched once synaptic vesicles are endocytosed and re-acidified, thus it reliably reflects the kinetics of endocytosis.

When neurons were stimulated with $300 \mathrm{APs}$ at $5 \mathrm{~Hz}$, the fluorescence intensity of individual $\mathrm{vGpH}$-expressing boutons in control neurons increased rapidly, reached a peak, and then decayed to the resting levels with an exponential time course while the kinetics of $\mathrm{vGpH}$ fluores- 


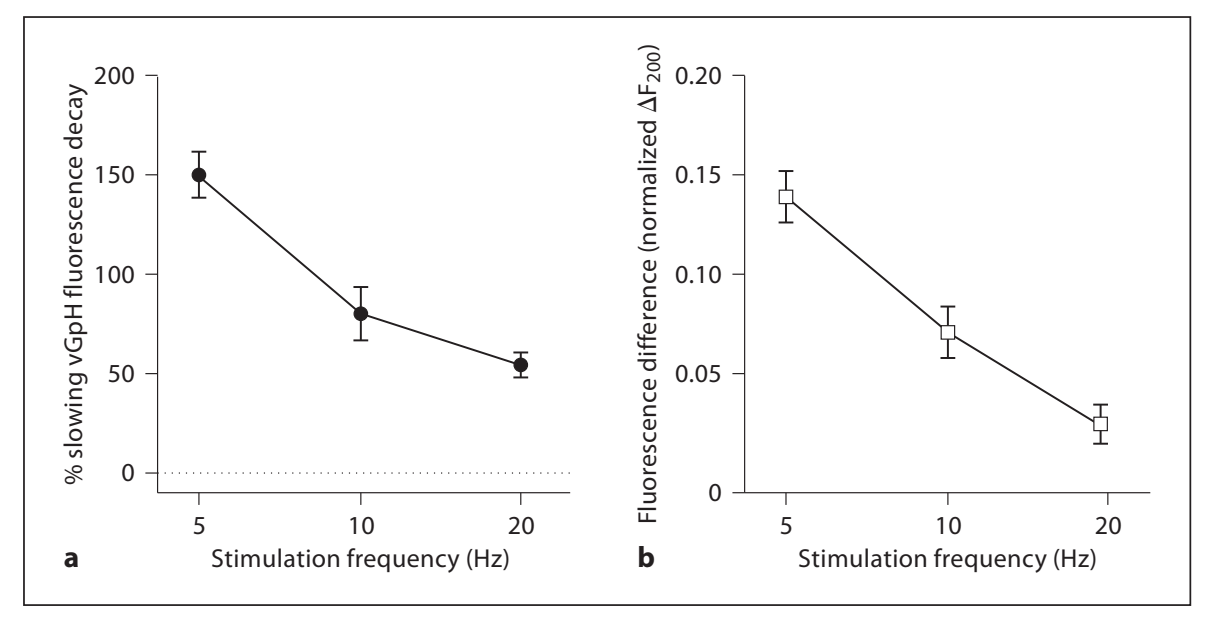

Fig. 4. The degree of endocytic defects is less pronounced at high frequency stimulations. a Time constant of Dyrk1A expressing boutons under various stimulation frequencies was normalized to that of control and expressed as percentage of slowing vGpH fluorescence decay. Dotted line indicates $0 \%$ slowing effect thus, no difference compared to control. The delay of synaptic vesicle turnover is more pronounced at low stimulation frequency than at high stimulation frequency (\% slowing effect: $150.8 \%$ at $5 \mathrm{~Hz}$, $80.8 \%$ at $10 \mathrm{~Hz}, 55.1 \%$ at $20 \mathrm{~Hz}) .4-5$ neurons were selected in each

cence decay was significantly slowed in boutons overexpressing Dyrk1A, reflecting a slower rate of synaptic vesicle endocytosis $(\tau=23.0 \pm 1.8 \mathrm{~s}$ for control; $\tau=56.7 \pm$ $5.7 \mathrm{~s}$ for Dyrk1A; fig. 3 and online supplementary table 1). Residual $\mathrm{vGpH}$ fluorescence $200 \mathrm{~s}$ after stimulation was much higher in Dyrk1A-expressing synapses compared to control synapses (fig. 3f). The overexpression of Dyrk1A-2YF again did not show any defects in synaptic vesicle endocytosis $(\tau=23.7 \pm 2.5 \mathrm{~s}$ for Dyrk1A-2YF; fig. 3 and online supplementary table 1). The endocytic defects by Dyrk1A overexpression were fully recovered to the control level by shRNA induced knock-down of Dyrk1A in Dyrk1A overexpression background (online supplementary figure 5).

We also challenged the neurons with higher stimulation frequencies, $10 \mathrm{~Hz}$ and $20 \mathrm{~Hz}$ (fig. 4 and online supplementary figures 6 and 7). Compared to $5 \mathrm{~Hz}$ stimulation, $10 \mathrm{~Hz}$ stimulation and $20 \mathrm{~Hz}$ stimulation showed less pronounced, although significant, endocytic delay with Dyrk1A overexpression (\% slowing effect: $150.8 \%$ at $5 \mathrm{~Hz}, 80.8 \%$ at $10 \mathrm{~Hz}$, and $55.1 \%$ at $20 \mathrm{~Hz}$; fig. $4 \mathrm{a}$ and online supplementary table 1). We also measured the residual $\mathrm{vGpH}$ fluorescence $200 \mathrm{~s}$ after stimulation at each stimulation frequency. Compared to $5 \mathrm{~Hz}$ stimulation frequency at which there is an incompletion of synaptic vesicle endocytosis $\sim 200 \mathrm{~s}$ after stimulation $(\sim 15 \%$ of independent experiment and 10-20 boutons from a single neuron were analyzed for each stimulus frequency. $\mathbf{b}$ The residual $\mathrm{vGpH}$ fluorescence at $200 \mathrm{~s}$ under various stimulation frequencies was normalized to that of the initial resting state of each presynaptic bouton. Incompletion of synaptic vesicle endocytosis $200 \mathrm{~s}$ after stimulation at $5 \mathrm{~Hz}$ stimulation frequency while at higher stimulation frequency $(10$ or $20 \mathrm{~Hz})$, synaptic vesicles were almost fully endocytosed. Data are presented as means $\pm \mathrm{SE}$.

vGpH fluorescence remained), at higher stimulation frequencies (10 and $20 \mathrm{~Hz}$ ), synaptic vesicles were almost fully recycled back to the cytosol $200 \mathrm{~s}$ after stimulation (less than $5 \%$ of $\mathrm{vGpH}$ fluorescence remained). Together, our results indicate that the delay of synaptic vesicle turnover is more pronounced at low stimulus frequency than at high stimulus frequency (fig. 4).

To further corroborate the effect of Dyrk1A expression on synaptic vesicle endocytosis in a more physiologically relevant DS model, we used Dyrk1A TG mice that contain additional extra copy of the human Dyrk1A gene in a bacterial artificial chromosome [3]. These TG mice show a 1.5-fold overexpression of Dyrk1A compared to control mice, which is a comparable amount of elevation in DS patients [24]. They also exhibit significant impairments in hippocampal-dependent memory tasks, hyperphosphorylation of tau and amyloid precursor protein similar to that is seen in DS patients [3].

We cultured hippocampal neurons from the TG mice, transfected with $\mathrm{vGpH}$, and performed endocytosis assay with $300 \mathrm{APs}$ at $5 \mathrm{~Hz}$. Consistent with overexpression results, synaptic vesicle endocytosis in TG mice-derived hippocampal neurons was significantly slowed compared to that in control (fig. 5). When we compared the first $50 \mathrm{~s}$ of recycling kinetics, control neurons endocytosed more than $\sim 80 \%$ of the exocytosed synaptic vesicles whereas TG 

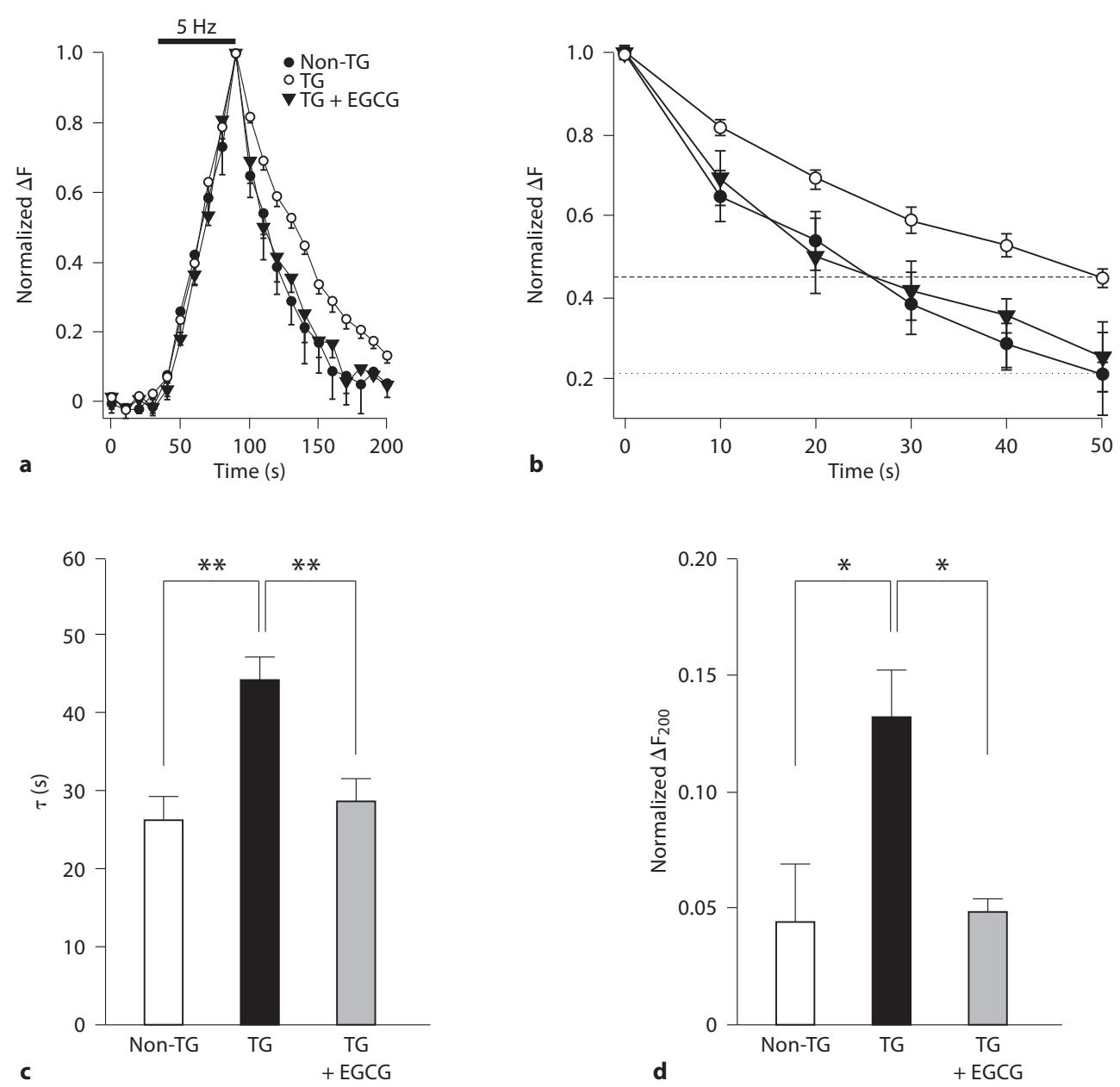

Fig. 5. Hippocampal neurons derived from Dyrk1A transgenic mice show the defects in synaptic vesicle endocytosis. Hippocampal neurons from Dyrk1A TG or control littermate were transfected with $\mathrm{vGpH}$ and treated with or without $1 \mu \mathrm{M}$ EGCG for $48 \mathrm{~h}$. a, c Average fluorescence intensity profiles were plotted as $\Delta \mathrm{F} / \mathrm{F}_{0}$ against time, following stimulation with 300 APs at $5 \mathrm{~Hz}$ (dark bar). The decay of $\mathrm{vGpH}$ fluorescence was fitted by a single exponential with $\tau=26.2 \pm 3.2 \mathrm{~s}(\mathrm{n}=4)$ for non-TG littermate; $\tau=44.4 \pm 2.9 \mathrm{~s}(\mathrm{n}=6)$ for Dyrk1A TG; $\tau=28.9 \pm 2.8 \mathrm{~s}(\mathrm{n}=4)$

mice-derived neurons still retained $\sim 45 \%$ of exocytosed synaptic vesicles at the plasma membrane (fig. 5b), indicating the significant delay in synaptic vesicle endocytosis.

To test whether the kinase activity of Dyrk1A is required for the endocytic defects observed in TG mice, we treated the neurons with EGCG or harmine, a specific Dyrk1A kinase inhibitor, for 2 days before the assay [2, $25,26]$. The endocytic defects in hippocampal neurons of TG mice was fully recovered with the treatment of EGCG for Dyrk1A TG with EGCG. Data were collected 4-5 neurons in each independent experiment, and 10-20 boutons from a single neuron were analyzed. b Expanded data collected within initial 50 s indicate the marked differences in the initial endocytic kinetics among neurons derived from non-TG mice, Dyrk1A TG mice, and Dyrk1A TG mice with EGCG. d The residual vGpH fluorescence intensity at $200 \mathrm{~s}$ was normalized to the initial resting state of each presynaptic bouton. Data are presented as means \pm SE. ${ }^{*} \mathrm{p}<0.05,{ }^{* *} \mathrm{p}<0.01$ (ANOVA and Tukey's HSD post hoc test).

$(\tau=26.2 \pm 3.2 \mathrm{~s}$ for non-TG mice; $\tau=44.4 \pm 2.9 \mathrm{~s}$ for Dyrk1A TG mice; $\tau=28.9 \pm 2.8$ s for EGCG treatment; fig. 5 and online supplementary table 1) as well as harmine $(\tau=27.3 \pm 4.1 \mathrm{~s}$ for non-TG mice; $\tau=41.7 \pm 3.3 \mathrm{~s}$ for Dyrk1A TG mice; $\tau=26.7 \pm 3.3 \mathrm{~s}$ for harmine treatment; online supplementary figure 8), indicating that elevated level of phosphorylation by overexpressed Dyrk1A in TG mice is attributed to the defects in synaptic vesicle endocytosis. 


\section{Discussion}

Dyrk1A is expressed in the cortex, spinal cord, and olfactory bulb, a pattern that correlates well with the regions profoundly affected in DS; thus, it is known to play a role in causing the mental retardation phenotype in DS [27, 28]. Indeed, Dyrk1A overexpression in TG mice consistently causes cognitive dysfunction $[3,29]$ although the underlying mechanism of the defects is largely unknown. Dyrk1A phosphorylates various proteins including several transcription factors such as cyclic AMP response element-binding protein (CREB), nuclear factor of activated $\mathrm{T}$ cells (NFAT), and forkhead in rhabdomyosarcoma (FKHR). Furthermore, overexpression of Dyrk1A causes splicing aberrations which induces the changes in the expression levels of various mRNAs such as TrkBT1, BDNF, AchE-S, AchE-R, neuroligin 1 and 3 [30]. All of these changes together may affect synaptogenesis and synaptic plasticity, leading to impairments in brain function.

Therefore, it is possible that Dyrk1A exerts its overdose effects on synaptic vesicle endocytosis by over-phosphorylating many different neural gene products other than endocytic proteins. COS-7 cells, however, do not express any neuron-specific genes, but still showed an endocytic defect by Dyrk1A overexpression and this can be reversed by kinase-dead Dyrk1A mutant expression. Furthermore, our TIRFM data suggested that Dyrk1A overexpression causes the defects in recruitment of key endocytic proteins in COS-7 cells. Despite the mechanistic differences between neurons and COS-7 cells, the basic principles that regulate clathrin-mediated endocytic process are common in both systems. Therefore, we believe that Dyrk1A's effect on endocytic process is mainly attributed to its phosphorylating activity toward endocytic proteins.

Interestingly, we found that using different stimulus frequency, the defects in synaptic vesicle turnover are more pronounced at low stimulus frequency than at high stimulus frequency. One possible explanation of these results is that at low stimulus frequency, limited fraction of the entire releasable pool may be used for recycling, thus could be more affected by Dyrk1A-directed phosphorylation. Another explanation is that dephosphorylation of endocytic proteins is activity-dependently regulated. Indeed, recent studies suggest that calcineurin-dependent dephosphorylation of endocytic proteins was observed at strong stimulation frequency $(>20 \mathrm{~Hz})$, but not at mild stimulation frequencies [31, 32]. Therefore, at high frequency stimulation, calciuneurin-mediated dephosphorylation of endocytic proteins may counteract Dyrk1A- mediated phosphorylation, attenuating the inhibitory effect of Dyrk1A on endocytosis.

Besides the TG mice we have used in the current study, two other Dyrk1A TG mice were known. TgDyrk1A contain a 6.7-kb fragment from sMT-Ia/Dyrk1A chimeric gene and 152F7 mice contain a fragment of yeast artificial chromosome containing DSCR genes, such as DSCR5, TTC, DSCR3, and Dyrk1A [29, 33, 34]. Although these TG mice models have provided invaluable information, each possesses pitfalls when used to investigate the sole function of Dyrk1A. The 152F7 mice contain 4 additional genomic genes in addition to Dyrk1A gene, and thus could not represent the pure Dyrk1A gene effects. While TgDyrk1A drives the Dyrk1A expression by the inducible heterologous sMT-Ia promoter; thus, their expression levels are variable. Meanwhile, the Dyrk1A TG mice used in the current study carry a single copy of bacterial artificial chromosome clone that contained only the complete human Dyrk1A genomic DNA fragment [3]. Furthermore, the Dyrk1A expression is consistent under its own endogenous human promoter, rendering 1.5 -fold overexpression compared to that of control mice.

Although the degree of endocytic defects in Dyrk1A TG mice was not as pronounced as that in overexpression of Dyrk1A, it is noteworthy that DSCR of human chromosome 21 contains other proteins such as synaptojanin and regulators of calcineurin 1 (RCAN1) $[11,35,36]$. We and others showed that synaptojanin is a key regulator of endocytosis, and its overexpression or deficiency caused severe endocytic defects $[37,38]$. RCAN1 is an inhibitor of calcineurin, a key phosphatase for endocytic proteins [39]. Therefore, additive effect of such proteins surely could cause severe defects in synaptic vesicle endocytosis, which possibly contributes to altered synaptic function such as cognitive impairment observed in DS patients. Further investigation of the interplay between Dyrk1A and other kinase/phosphatase in the presynaptic terminals will help in development of novel therapeutic strategies to prevent or inhibit synaptic dysfunctions in DS and thus treat the mental retardation in this syndrome.

\section{Acknowledgements}

This research was supported by a grant of the Korea Healthcare technology R\&D Project (A084393) to S.C. from the Ministry for Health, Welfare \& Family Affairs, Republic of Korea. Confocal and TIRF microscopy data for this study were acquired and analyzed in the Biomedical Imaging Center at Seoul National University College of Medicine. 


\section{References}

$>1$ Korenberg JR, et al: Down syndrome phenotypes: the consequences of chromosomal imbalance. Proc Natl Acad Sci USA 1994;91: 4997.

12 Liu F, et al: Overexpression of Dyrk1A contributes to neurofibrillary degeneration in Down syndrome. FASEB J 2008;22:3224.

$\checkmark 3$ Ahn KJ, et al: DYRK1A BAC transgenic mice show altered synaptic plasticity with learning and memory defects. Neurobiol Dis 2006;22:463.

$>4$ Fujita H, et al: Microdeletion of the Down syndrome critical region at 21q22. Am J Med Genet A 2010;152A:950.

5 Alvarez M, Estivill X, de la Luna S: DYRK1A accumulates in splicing speckles through a novel targeting signal and induces speckle disassembly. J Cell Sci 2003;116:3099.

$\checkmark 6$ Marti E, et al: Dyrk1A expression pattern supports specific roles of this kinase in the adult central nervous system. Brain Res 2003;964:250.

7 Murakami N, et al: Phosphorylation of amphiphysin I by minibrain kinase/dual-specificity tyrosine phosphorylation-regulated kinase, a kinase implicated in Down syndrome. J Biol Chem 2006;281:23712.

$>8$ Chen-Hwang MC, Chen HR, Elzinga M, Hwang YW: Dynamin is a minibrain kinase/ dual specificity Yak1-related kinase 1A substrate. J Biol Chem 2002;277:17597.

$>9$ Adayev T, Chen-Hwang MC, Murakami N, Wang R, Hwang YW: MNB/DYRK1A phosphorylation regulates the interactions of synaptojanin 1 with endocytic accessory proteins. Biochem Biophys Res Commun 2006;351:1060.

10 Murakami N, Bolton D, Hwang YW: Two nonmuscle myosin II heavy chain isoforms expressed in rabbit brains: filament forming properties, the effects of phosphorylation by protein kinase $\mathrm{C}$ and casein kinase II, and location of the phosphorylation sites. Biochemistry 2009;48:9297.

$\checkmark 11$ Cremona O, et al: Assignment of SYNJ1 to human chromosome 21q22.2 and Synj12 to the murine homologous region on chromosome 16C3-4 by in situ hybridization. Cytogenet Cell Genet 2000;88:89.

$\checkmark 12$ Arai Y, Ijuin T, Takenawa T, Becker LE, Takashima S: Excessive expression of synaptojanin in brains with Down syndrome. Brain Dev 2002;24:67.

13 Voronov SV, et al: Synaptojanin 1-linked phosphoinositide dyshomeostasis and cognitive deficits in mouse models of Down's syndrome. Proc Natl Acad Sci USA 2008; 105:9415.
14 Slepnev VI, Ochoa GC, Butler MH, Grabs D, De Camilli P: Role of phosphorylation in regulation of the assembly of endocytic coat complexes. Science 1998;281:821.

15 Cousin MA, Robinson PJ: The dephosphins: dephosphorylation by calcineurin triggers synaptic vesicle endocytosis. Trends Neurosci 2001;24:659.

16 Cousin MA, Tan TC, Robinson PJ: Protein phosphorylation is required for endocytosis in nerve terminals: potential role for the dephosphins dynamin I and synaptojanin, but not AP180 or amphiphysin. J Neurochem 2001;76:105-116.

17 Chang S, De Camilli P: Glutamate regulates actin-based motility in axonal filopodia. Nat Neurosci 2001;4:787.

$>18$ Xia Z, Dudek H, Miranti CK, Greenberg ME: Calcium influx via the NMDA receptor induces immediate early gene transcription by a MAP kinase/ERK-dependent mechanism. J Neurosci 1996;16:5425.

19 Huang Y, et al: Mnb/Dyrk1A phosphorylation regulates the interaction of dynamin 1 with $\mathrm{SH} 3$ domain-containing proteins. Biochemistry 2004;43:10173.

20 Soulet F, Yarar D, Leonard M, Schmid SL: SNX9 regulates dynamin assembly and is required for efficient clathrin-mediated endocytosis. Mol Biol Cell 2005; 16:2058.

-21 Rappoport JZ: Focusing on clathrin-mediat ed endocytosis. Biochem J 2008;412:415.

22 Perera RM, Zoncu R, Lucast L, De Camilli P, Toomre D: Two synaptojanin 1 isoforms are recruited to clathrin-coated pits at different stages. Proc Natl Acad Sci USA 2006;103: 19332.

23 Voglmaier SM, et al: Distinct endocytic pathways control the rate and extent of synaptic vesicle protein recycling. Neuron 2006; 51:71.

24 Antonarakis SE, Lyle R, Dermitzakis ET, Reymond A, Deutsch S: Chromosome 21 and down syndrome: from genomics to pathophysiology. Nat Rev Genet 2004;5:25.

25 Adayev T, Chen-Hwang MC, Murakami N, Wegiel J, Hwang YW: Kinetic properties of a MNB/DYRK1A mutant suitable for the elucidation of biochemical pathways. Biochemistry 2006;45:12011

26 Gockler N, et al: Harmine specifically inhibits protein kinase DYRK1A and interferes with neurite formation. FEBS J 2009;276: 6324.
27 Kola I, Hertzog PJ: Down syndrome and mouse models. Curr Opin Genet Dev 1998; $8: 316$.

28 Guimera J, et al: A human homologue of Drosophila minibrain (MNB) is expressed in the neuronal regions affected in Down syndrome and maps to the critical region. Hum Mol Genet 1996;5:1305.

29 Altafaj X, et al: Neurodevelopmental delay, motor abnormalities and cognitive deficits in transgenic mice overexpressing Dyrk1A (minibrain), a murine model of Down's syndrome. Hum Mol Genet 2001;10:1915.

-30 Toiber D, et al: Engineering DYRK1A overdosage yields Down syndrome-characteristic cortical splicing aberrations. Neurobiol Dis 2010;40:348.

31 Clayton EL, Evans GJ, Cousin MA: Activitydependent control of bulk endocytosis by protein dephosphorylation in central nerve terminals. J Physiol 2007;585:687.

32 Clayton EL, et al: The phospho-dependent dynamin-syndapin interaction triggers activity-dependent bulk endocytosis of synaptic vesicles. J Neurosci 2009;29:7706.

33 Smith DJ, et al: Functional screening of $2 \mathrm{Mb}$ of human chromosome 21q22.2 in transgenic mice implicates minibrain in learning defects associated with Down syndrome. Nat Genet 1997; 16:28

-34 Smith DJ, Zhu Y, Zhang J, Cheng JF, Rubin EM: Construction of a panel of transgenic mice containing a contiguous $2-\mathrm{Mb}$ set of YAC/P1 clones from human chromosome 21q22.2. Genomics 1995;27:425.

35 Fuentes JJ, Pritchard MA, Estivill X: Genomic organization, alternative splicing, and expression patterns of the DSCR1 (Down syndrome candidate region 1) gene. Genomics 1997;44:358.

$>36$ Cheon MS, et al: Protein levels of genes encoded on chromosome 21 in fetal Down syndrome brain: challenging the gene dosage effect hypothesis. Part III. Amino Acids 2003; 24:127.

37 Kim S, et al: Regulation of transferrin recycling kinetics by PtdIns[4,5]P2 availability. FASEB J 2006;20:2399.

38 Mani M, et al: The dual phosphatase activity of synaptojanin 1 is required for both efficient synaptic vesicle endocytosis and reavailability at nerve terminals. Neuron 2007; 56:1004.

39 Fuentes JJ, et al: DSCR1, overexpressed in Down syndrome, is an inhibitor of calcineurin-mediated signaling pathways. Hum Mol Genet 2000;9:1681. 